'Departamento de Psiquiatría,

Hospital Clínico, Facultad de

Medicina, Universidad de Chile, Santiago, Chile.

${ }^{2}$ CEMERA, Facultad de Medicina, Universidad de Chile, Santiago, Chile.

aPsicóloga, estudiante de Doctorado en Psicoterapia, becaria CONICYT, Pontificia Universidad Católica de Chile. Investigador-Docente Facultad de Psicología, Universidad del Desarrollo, Santiago, Chile.

${ }^{b}$ Doctora en Psicoterapia.

Financiado por Proyecto NS 100018 "Intervención Psicológica y Cambio en Depresión", de la Iniciativa Científica Milenio del Ministerio de Economía, Fomento y Turismo.

Recibido el 31 de enero de 2013, aceptado el 4 de septiembre de

Correspondencia a: Dra. Graciela Rojas Castillo Clínica Psiquiátrica, Hospital Clínico, Universidad de Chile

Av. La Paz 1003, Recoleta. Teléfono: 29788601

graciela.rojas.castillo@gmail.com

\section{Prevención, detección, tratamiento o seguimiento en salud mental de adolescentes a través del uso de Internet: una revisión sistemática cualitativa}

\author{
MARIANELA HOFFMANNª, GRACIELA ROJAS ${ }^{1}$, VANIA MARTÍNEZ ${ }^{2, b}$
}

\section{Use of Internet for mental health programs aimed at adolescents}

Background: The widespread use of INTERNET by adolescents, renders it a simple and accessible means for health care campaigns Aim: To perform a systematic review of ISI publications about INTERNET use for adolescent mental health prevention, detection, treatment or follow up. Material and Methods: Systematic reviews, meta analyses and controlled clinical trials in all languages were searched. Manuscripts without an abstract or only publishing the study design were excluded. All selected articles were critically read. Results: One hundred and five papers were found and 61 were excluded because they did not address the research question. Of the resting 44, only six complied with the search criteria among then, 3 articles correspond to systematic reviews addressing early intervention programs and mental hearth treatment based on Internet; the other 3 articles correspond to controlled clinical trials addressing treatment interventions of anxiety and depression and 1 address on depression prevention program. Conclusions: INTERNET facilitates the application of mental health promotion, prevention and intervention among adolescents. However the resource is still underused.

(Rev Med Chile 2014; 142: 494-500)

Key words: Adolescent; Internet; Mental Health. $\checkmark$ n la adolescencia los problemas de salud mental son muy comunes. Se ha reportado que uno de cada diez adolescentes presenta problemas de salud mental graves, lo cual afecta su desempeño en la casa, escuela o comunidad ${ }^{1}$.

En Chile, Vicente y cols (en el año 2012) realizaron un estudio de prevalencia de trastornos psiquiátricos presentes en los últimos 12 meses basado en el DSM-IV, utilizando una muestra nacional representativa de niños (4 a 11 años) y adolescentes (12 a 18 años). Encontraron una prevalencia de los trastornos psiquiátricos de $38,3 \%$ sin impedimento, la cual se ajusta a 22,5\% al aplicar criterios de discapacidad. Es decir, 22,5\% del total de niños y adolescentes chilenos presentan un diagnóstico con un nivel de compromiso clínicamente significativo que requiere de una intervención especializada de salud mental. Específicamente en adolescentes la prevalencia de trastornos psiquiátricos en los últimos doce meses con impedimento es de $16,5 \%{ }^{2}$.

$\mathrm{Si}$ bien la prevalencia de problemas graves de salud mental es alta en la población infantojuvenil, entre $75 \%$ y $80 \%$ de los que necesitan atención en salud mental, no reciben este servicio ${ }^{3}$. En Chile, del total de niños y adolescentes con trastornos psiquiátrico con impedimento $(\mathrm{n}=$ 329 ), sólo $19,1 \%$ de ellos recurrió a un servicio de salud mental formal ${ }^{2}$.

Nos encontramos entonces con una alta necesidad de atención en salud mental en la adolescencia, pero con escaso acceso a tratamiento. Así, muchos adolescentes no reciben los servicios de atención que son necesarios. Se han documentado 
variados motivos de por qué ocurre esto, entre ellos se encuentra la preocupación de los adolescentes por la confidencialidad en la atención. En Estados Unidos de Norteamérica los adolescentes que más evitan la asistencia a servicios de salud por la preocupación de la confidencialidad, son los más vulnerables, presentando cuadros depresivos, pensamientos suicidas, abuso de drogas y/o alcohol ${ }^{4}$.

Existe una gran brecha entre las necesidades y el uso de servicios de salud por parte de los adolescentes. De hecho, se han identificado las principales barreras que tienen que afrontar en diferentes partes del mundo para acceder a los servicios de atención primaria. Entre éstas se encuentran la falta de servicios cercanos; existencia de leyes y políticas restrictivas que dificultan el acceso a servicios; altos costos (por lejanía); horarios poco convenientes; también la falta de conocimiento acerca la disponibilidad de los servicios existentes (por falta de información) ${ }^{5}$.

De acuerdo a lo descrito, Internet se convierte en una alternativa para afrontar las barreras identificadas, al tratarse de un medio de acceso conveniente, confidencial y cercano a los jóvenes para obtener información de salud mental. Y si bien Internet no remplaza a otras fuentes de información tales como pares y adultos, puede ser un gran aporte .

De acuerdo a la "Encuesta sobre Acceso, Uso y Usuarios de Internet Banda Ancha en Chile", $60,5 \%$ de los hogares cuenta con un computador o notebook ${ }^{7}$. A su vez, la Encuesta CASEN 2006 estableció que la penetración de computadores era de $39 \%$ en poco más de 3 años, produciéndose un importante aumento en el acceso a este recurso tecnológico ${ }^{8}$. Por otro lado, el acceso a Internet alcanza al año 2009 a 40,6\% de los hogares encuestados ${ }^{7}$.

En la sexta encuesta nacional de juventud del Instituto Nacional de la Juventud, se observó que $56,1 \%$ de los jóvenes utiliza Internet "todos o casi todos los días", lo cual representa un incremento de 13,7 puntos porcentuales en relación a la medición del año 2006, tendencia de incremento que se viene observando desde el año 2003. El 53,5\% de los jóvenes declara acceder a Internet desde su hogar, seguido por $32,4 \%$ que señala hacer lo mismo desde un cibercafé; y en tercer lugar se ubican los establecimientos educacionales con 19,8\% .

Esta evidencia da cuenta de la alta accesibilidad que tienen los adolescentes al uso de Internet, teniendo la posibilidad de acceder a temáticas de salud mental a través de este medio en sus hogares, en escuelas, liceos, centros de formación técnica y consultorios de atención primaria que cuentan con Internet.

Dado lo anterior surge como pregunta ¿Cómo el uso de Internet puede ayudar a la prevención, detección, tratamiento o seguimiento en salud mental de adolescentes?

El objetivo de la presente comunicación es dar a conocer los resultados de una revisión sistemática cualitativa que buscó identificar y analizar literatura sobre el uso de Internet en la prevención, detección, tratamiento o seguimiento en salud mental de adolescentes.

\section{Método}

Se llevó a cabo una revisión sistemática cualitativa que buscó revisiones sistemáticas, metaanálisis y ensayos clínicos controlados (ECC) en todos los idiomas, que utilizaran Internet como recurso para la prevención, detección, tratamiento o seguimiento en salud mental de adolescentes.

Se buscaron artículos que cumplieran con la categoría ISI, para lo cual se revisó la base de datos Web of Knowledge con las palabras claves "adolescence" AND "mental health" AND "Internet". La búsqueda incluyó todos los idiomas de publicación y se concentró desde enero de 2001 hasta el 12 de diciembre de 2011.

Para cada uno de los artículos seleccionados se realizó una evaluación crítica de la literatura, excluyéndose artículos que no tuviesen resúmenes o que publicasen el diseño de un estudio sin sus resultados.

\section{Resultados}

Se encontraron 105 artículos, de los cuales se revisaron los títulos y resúmenes para evaluar si correspondían a la temática de la revisión, excluyéndose 61 por no responder al objetivo de la revisión. Luego se procedió a leer los 44 artículos preseleccionados, de los cuales se eliminaron 38 artículos por no cumplir con los criterios de inclusión de la revisión, quedando 6 artículos que cumplieron con los criterios de la búsqueda.

De los 6 artículos seleccionados, 3 corresponden a ECC y 3 a revisiones sistemáticas, no encontrándose meta-análisis en la búsqueda. En la Figura 1 se puede observar el flujo de la 


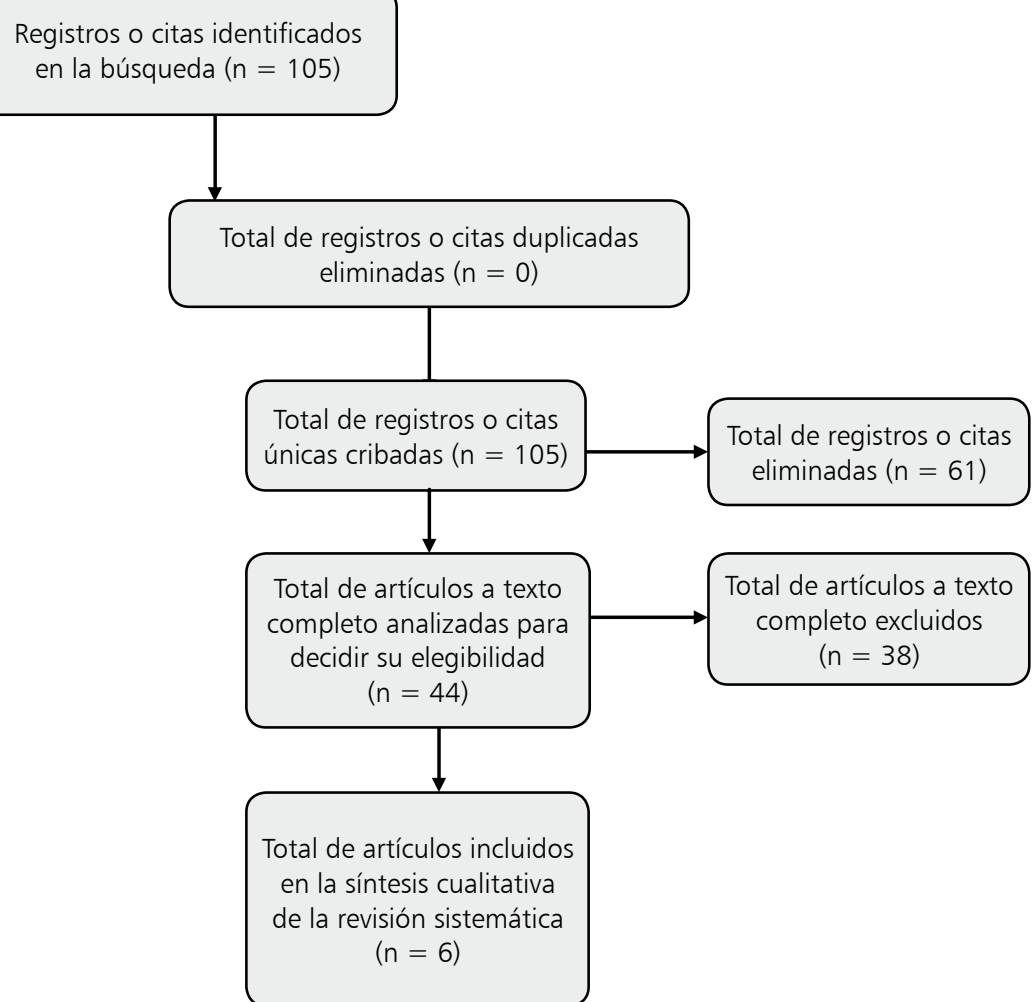

Figura 1. Diagrama de flujo de la información a través de las diferentes fases de la revisión sistemática cualitativa

información a través de las diferentes fases de la revisión realizada.

En relación a las revisiones sistemáticas, éstas abordan programas de intervención temprana y de tratamiento en salud mental basadas en Internet. El artículo de Benjamin Van Voorhees y cols (2011) aborda intervenciones para la prevención de depresión ${ }^{10}$, el artículo de Alison Calear y Helen Christensen (2010) revisa programas para la prevención de ansiedad y depresión ${ }^{11}$; y por último en artículo de Christopher Siemer y cols (2011) aborda programas de promoción de salud mental, intervenciones de prevención y tratamiento de diferentes problemas de salud mental ${ }^{12}$.

Principalmente los artículos se centran en tratamientos para la depresión (Ej., proyecto CATCH-IT, programa Mood GYM), ansiedad (ej., programa BRAVE Online), trastornos de alimentación (ej., programa My Body, My Life, The Student Bodiesprogram) y abuso de sustancias (ej., The Climate Schools program, entre otros $)^{10-12}$.

Es importante mencionar que la minoría de los programas señalados que se encontraron en las revisiones sistemáticas cuentan con ECC.

Algunos programas reclutan sus participantes en el contexto de atención primaria (primera entrevista y evaluación) y luego requieren acceso a Internet en el hogar para continuar con el programa. Estos programas son apoyados por profesionales de la salud, ya sea dentro del entorno médico o de forma remota por correo electrónico o teléfono. Otros programas realizan la primera entrevista y evaluación en establecimientos educacionales, y se imparten en éstos mismos. Una minoría de los programas se ofrece a través de un acceso abierto en sitio web desde su inicio (evaluación se realiza a través de Internet también $)^{10-12}$.

En la mayoría de los estudios se presta apoyo en línea a los usuarios de las intervenciones, pero los métodos varían considerablemente. Este apoyo es realizado por teléfono, a través de correos electrónicos o en el contexto escolar es realizado por un supervisor profesional (profesor). Por último en algunos no existe este soporte adicional. 
El apoyo adicional sirve para varios propósitos: monitorea el progreso del usuario en el sitio, revisa tareas y actividades de los programas, ofrece clases semanales presenciales, determina si existe riesgo en los participantes (aumento significativo de sintomatología, ideación suicida), velando así por la seguridad emocional de los usuarios; y por último, motiva la permanencia de los adolescentes en los programas ${ }^{10-12}$.

Los programas que se mencionan en estas revisiones se basan principalmente en la terapia cognitivo conductual (TCC), sólo uno de ellos señala incluir además la activación conductual y la terapia interpersonal. Todos son dirigidos tanto a hombres como a mujeres, participando en general mayoritariamente mujeres. Estos programas tienen una duración que fluctúa entre 5 y 14 sesiones y la mayoría cuenta con sistemas de seguimiento que oscila en una duración de dos meses a un año ${ }^{10-12}$.

En relación a los artículos de ECC, dos de éstos corresponden a artículos que abordan intervenciones de tratamiento y uno aborda un programa de prevención.

El artículo de Susan Spence y cols (2011) trata de un ECC que testea un tratamiento para la ansiedad denominado BRAVE for Teenagers-ONLINE (BO). Este programa fue diseñado para tratar fobia social, trastorno de ansiedad generalizada, ansiedad por separación y fobia específica en adolescentes por Internet. Está basado en la TCC, y consiste en 10 sesiones semanales para los adolescentes, dos sesiones de refuerzo que se realizan 1 y 3 meses después de la intervención, y cinco o seis sesiones para padres. El programa presenta información sobre manejo de la ansiedad, reconocimiento de los síntomas fisiológicos de la ansiedad, la exposición graduada y técnicas de resolución de problemas. La intervención incluye material de lectura, preguntas, ejercicios, juegos y concursos. Los usuarios completan la intervención a su propio ritmo a través de la página web del programa, con un breve contacto con un terapeuta, consistente en un correo electrónico semanal, quien revisa las tareas y actividades de la sesión. El terapeuta también colabora en el desarrollo de una jerarquía de exposición por correo electrónico o por teléfono ${ }^{13}$.

El ECC compara tres grupos, un grupo de tratamiento con $\mathrm{BO}$, un grupo al cual se le aplicó un programa equivalente al online, pero realizado cara a cara (CLIN) y un grupo control de participantes en lista de espera (WLC). En la evaluación a las 12 semanas posteriores a la evaluación basal, se mostraron reducciones significativamente mayores en los diagnósticos de ansiedad y en los síntomas de ansiedad para los grupos BO y CLIN, en comparación con el WLC. Estas mejoras se mantienen o mejoran aun más para ambas condiciones, con diferencias mínimas entre ellas, a los 6 y 12 meses de seguimiento. Se concluye en el estudio que la entrega en línea de la TCC con apoyo mínimo de un terapeuta, es igual de eficaz que la terapia cara a cara en el tratamiento de los trastornos de ansiedad en los adolescentes ${ }^{13}$.

El artículo de Paul Stallard y cols (2011) trata de una intervención denominada Think, Feel, Do (TFD). Este programa basado en la TCC tiene como objetivo el tratamiento de depresión (leve o moderada) y ansiedad (trastorno de ansiedad generalizada, fobias específicas, fobia social y trastorno de pánico) en adolescentes. Está compuesto por seis sesiones de 30 a 45 min de duración que se desarrollan en un CD-ROM y son facilitadas presencialmente por un profesional entrenado (psicólogo, profesor o enfermera) en su totalidad. Se enseñan temáticas tales como el reconocimiento de emociones, conexión entre pensamientos y emociones, aprendizaje sobre cómo cambiar pensamientos negativos a positivos, controlar emociones desagradables y resolución de problemas. El programa es interactivo, con respuestas a las pruebas y ejercicios que se introducen en el programa y se guardan estas respuestas para poder ser revisadas por el facilitador. El programa es multimedia e incluye sonidos, fotos, dibujos animados y música. Al final de cada sesión los usuarios reciben una breve tarea ${ }^{14}$.

El ECC compara dos grupos, un grupo donde se aplicó la intervención TFD y un grupo control en lista de espera para tratamiento. En los análisis de muestras pareadas se demostraron mejoras significativas en 3 sub-escalas en la condición de control, en comparación con 7 sub-escalas en el grupo TFD. De acuerdo a los comentarios de los participantes se demostró moderada a alta satisfacción de éstos en el programa. Se concluye en el artículo que el ECC proporciona resultados preliminares, pero alentadores para la eficacia y la aceptabilidad de la intervención TFD en adolescentes con la sintomatología depresiva y ansiosa ${ }^{14}$.

Y por último, el artículo de Benjamin Van Voorhees y cols (2009) trata de una intervención preventiva de depresión. Proyecto CATCH-IT (PC) es un programa gratuito de entrenamiento 
por Internet que se basa en la activación conductual, TCC y la psicoterapia interpersonal. El programa tiene como objetivo enseñar a los adolescentes a reducir comportamientos que aumentan su vulnerabilidad a los trastornos depresivos (la rumiación, las evaluaciones negativas) e incrementar los comportamientos protectores (el apoyo social, la programación conductual). El programa consta de 14 módulos e incluye explicaciones de los principales conceptos, historias de adolescentes, ejercicios de desarrollo de habilidades, retroalimentación y recompensas. El sitio web interactúa con los usuarios a través de tareas y narraciones. PC se difunde en el ámbito de la atención primaria y se ha evaluado comparando diferentes niveles de apoyo profesional ${ }^{15}$.

En el ECC los adolescentes fueron asignados dos grupos: Consejería breve (1-2 min) + PC, en comparación con una entrevista motivacional (10-15 min $)+$ PC. En el artículo se presenta la comparación de las medidas de participación y satisfacción para los dos grupos considerando un mínimo de 12 meses después de la inscripción. Los autores concluyen que utilizar una entrevista motivacional puede aumentar la dosis de uso del programa de intervención por Internet ${ }^{15}$ (Tabla 1 para la revisión de las variables y principales resultados).

\section{Discusión}

Existe abundante literatura sobre el uso de Internet en la adolescencia y su relación con la salud mental. Sin embargo, el foco de estas publicaciones no es hacia la prevención, detección, tratamiento y/o seguimiento de salud mental, sino más bien tienen relación con los efectos nocivos que podría tener el uso de Internet en la salud mental en este grupo etario, por diferentes motivos, ya sea adicción al uso de Internet, adicción a los vídeo juegos en línea, efectos nocivos de los vídeo juegos, acoso por Internet, etc. De los artículos que realmente apuntan a la dirección de esta revisión, son muy escasos los que cumplen con los criterios de calidad inicialmente establecidos para poder ingresar a la revisión sistemática, quedando de manifiesto la necesidad de desarrollar más estudios en esta área que cuenten con rigurosidad metodológica.

Los artículos encontrados en la presente revisión se centran en programas de prevención y tratamiento de trastornos mentales comunes en la adolescencia, como la depresión y ansiedad principalmente, y sólo en uno de ellos también se aborda los trastornos de alimentación y abuso de sustancias. La totalidad de los programas revisados se basan principalmente en la TCC y tienden a combinar los programas por Internet con otras formas de contacto con los usuarios, como entrevistas presenciales iniciales, contacto telefónico o correos electrónicos durante las intervenciones o en los seguimientos. Las intervenciones usan una amplia gama de experiencias multimedia, incluyendo juegos, música, vídeos, historias, fotografías, realidad virtual. Y en general realizan un proceso de seguimiento que oscila entre dos meses a un año de duración.

Los artículos encontrados corresponden a publicaciones de los últimos 3 años (2009 al 2011) que se han publicado en países de habla inglesa, dando cuenta que el uso de Internet como recurso para la prevención y tratamiento de salud mental en adolescentes es de interés reciente y de desarrollo incipiente. Sin embargo, se reportan importantes ventajas del uso de este recurso en los programas de salud mental.

Entre las ventajas del uso de Internet en los programas de promoción e intervención de salud mental destacan el que facilita el acceso de estos programas en zonas rurales donde se cuenta con menores posibilidades de acceder a un tratamiento cara a cara; es más económico ya que se ahorran los costos de traslados; otorga flexibilidad a los usuarios ya que éstos pueden acceder a las sesiones en cualquier momento según sus propias disponibilidades de horarios; mantiene el anonimato y confidencialidad de los usuarios con lo cual se evita la dificultad de sentirse estigmatizados por asistir a sesiones con un profesional de salud mental. Todas estas ventajas permiten a los adolescentes evadir las barreras que se han identificado tradicionalmente para acceder a los servicios de salud. Además, si bien la población adolescente es compleja en cuanto a la motivación que presenta para acceder a servicios de salud mental, es importante tener presente que la población joven ha sido denominada como la generación de los nativos digitales por ser quienes muestran el uso más intensivo de estas herramientas en su vida cotidiana. Las incorporan como un elemento de base que les facilita la inserción en diversos ámbitos que componen la vida social ${ }^{9}$. En este escenario Internet puede ser una vía de entrada a la salud mental para los adolescentes, siendo un recurso cercano a ellos y de uso frecuente. 


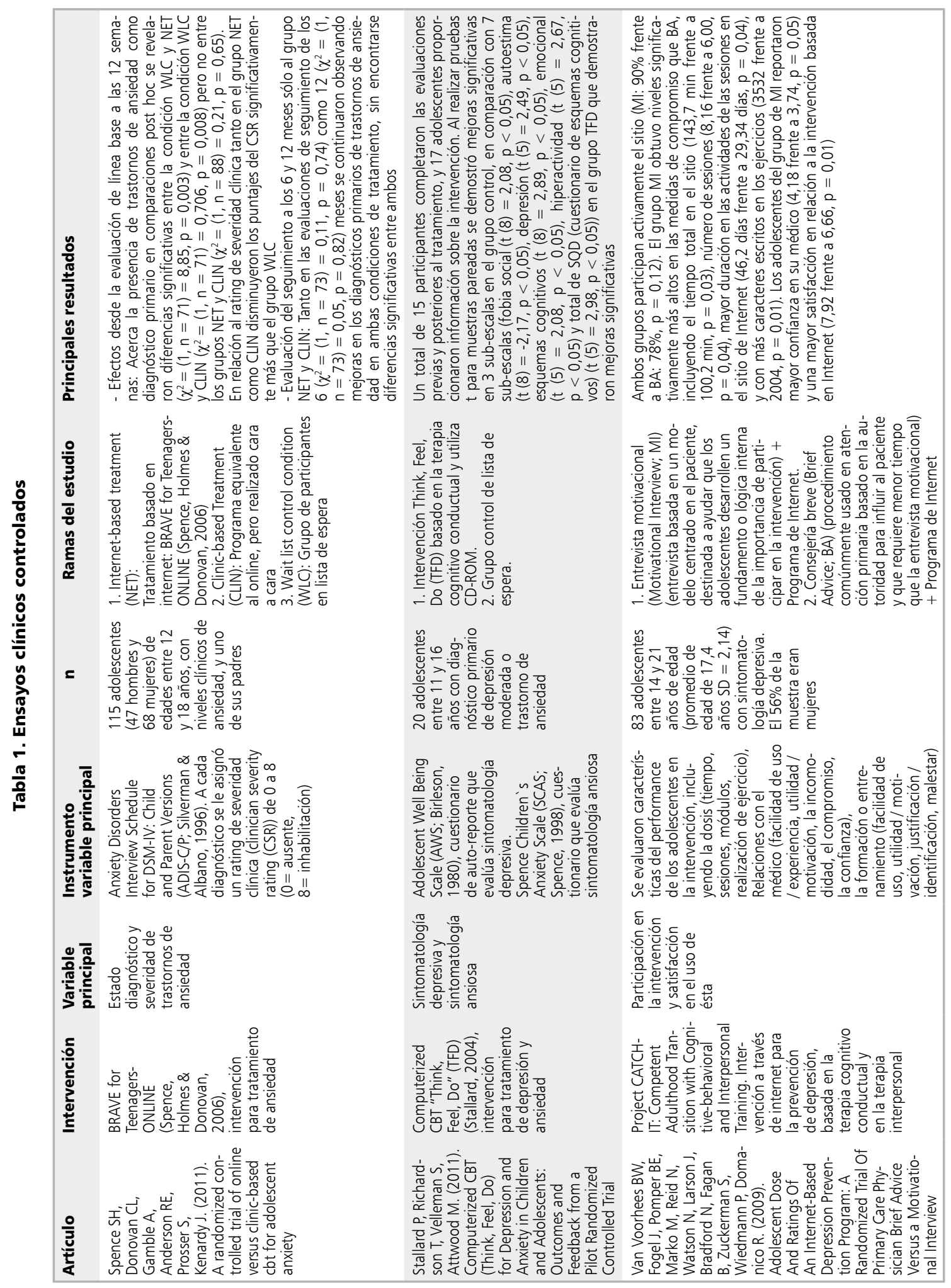


Uso de Internet en programas de salud mental para adolescentes - M. Hoffmann et al

Los beneficios no son sólo para los usuarios, sino que también para quienes están a cargo de los programas de salud mental, ya que pueden otorgar atenciones a poblaciones que no se accede fácilmente a través de la atención cara a cara; se trata de intervenciones económicas ya que el gasto de funcionamiento de las aplicaciones en Internet es más bajo que la tradicional atención cara a cara ${ }^{11}$ y por último facilita el seguimiento y monitoreo de los progresos de los usuarios.

Considerando los importantes beneficios que otorga el uso de Internet en los programas de prevención y tratamiento de salud mental, resalta la necesidad de mayor desarrollo de estos programas e investigaciones, específicamente en adolescentes, dado que en la población adulta se encuentra más avanzado $^{11,14}$.

Dada las altas tasas de prevalencia de trastornos mentales que presenta la población infanto-juvenil y la cobertura de Internet chilena, sería de gran utilidad contar a futuro con estudios nacionales que incorporen esta tecnología en programas de salud mental para adolescentes, considerando sus particularidades socioculturales.

\section{Referencias}

1. New Freedom Commission on Mental Health. Achieving the promise: Transforming mental health care in America. Final report (DHHS Pub. No. SMA-03-3832). Rockville, MD: U.S. Department of Health and Human Services, Substance Abuse and Mental Health Services Administration, 2003. Disponible en: www.mentalhealthcommission.gov/reports/reports.htm [Consultado el 10 de octubre de 2012].

2. Vicente B, Saldivia S, De La Barra F, Kohn R, Pihan R, Valdivia $\mathrm{M}$, et al. Prevalence of child and adolescent mental disorders in Chile: a community epidemiological study. J Child Psychol Psychiatry 2012; 53 (10): 1026-35.

3. Kataoka S, Zhang L, Wells K. Unmet need for mental health care among U.S. children: Variation by ethnicity and insurance status. Am J Psychiatry 2002; 159 (9): 1548-55.

4. Lehrer JA, Pantell R, Tebb K, Shafer MA. Forgone Health Care among U.S. Adolescents: Associations between Risk Characteristics and Confidentiality Concern. J Adolesc Health 2007; 40: 218-26.

5. Tylee A, Haller DM, Graham T, Churchill R, Sanci LA. Youth-friendly primary-care services: how are we doing and what more needs to be done? The Lancet 2007; 369 (9572): 1565-73.

6. Gray NJ, Klein JD, Noyce PR, Sesselberg TS, Cantrill JA. Health information-seeking behaviour in adolescence: the place of the internet. Soc Sci Med 2005; 60 (7): 146778.

7. Observatorio Social de la Universidad Alberto Hurtado y Subsecretaría de Telecomunicaciones. Encuesta sobre Acceso, Uso y Usuarios de Internet Banda Ancha en Chile, 2009, Santiago, Chile. Disponible en: www.mtt.gob.cl/prontus_mtt/site/artic/20100427/ pags/20100427160001.html [Consultado el 25 de octubre de 2012].

8. Tecnología de Información y Comunicaciones, Encuesta CASEN 2006, Ministerio de Desarrollo Social, 2006, Santiago, Chile. Disponible en: www.ministeriodesarrollosocial.gob.cl/casen [Consultado el 29 de octubre de 2012].

9. Instituto Nacional de la Juventud (INJUV), Gobierno de Chile. Sexta Encuesta Nacional de la Juventud, 2010, Santiago, Chile. Disponible en:www.injuv.gob.cl/portal/ wp-content/ [Consultado el 7 de junio de 2013].

10. Van Voorhees BW, Mahoney N, Mazo R, Barrera AZ, Siemer CP, Gladstone T, et al. Internet-Based Depression Prevention over the Life Course: A Call for Behavioral Vaccines. Psychiatr Clin North Am 2011; 34 (1): 167-83.

11. Calear AL, Chistensen H. Review of internet-based prevention and treatment programs for anxiety and depression in children and adolescents. MJA 2010; 192 (11): 12-4.

12. Siemer CP, Fogel J, Van Voorhees BW. Telemental Health and Web-based Applications in Children and Adolescents. Child Adolesc Psychiatr Clin N Am 2011; 20 (1): 135-53.

13. Spence SH, Donovan CL, March S, Gamble A, Anderson RE, Prosser S, et al. A Randomized Controlled Trial of Online Versus Clinic-Based CBT for Adolescent Anxiety. J Consult Clin Psychol 2011; 79 (5): 629-42.

14. Stallard P, Richardson T, Velleman S, Attwood M. Computerized CBT (Think, Feel, Do) for Depression and Anxiety in Children and Adolescents: Outcomes andFeedback from a Pilot Randomized Controlled Trial. Behavioural and Cognitive Psychotherapy 2011; 39 (3): 273-84.

15. Van Voorhees BW, Fogel J, Pomper BE, Marko M, Reid $\mathrm{N}$, Watson N, et al. Adolescent Dose And Ratings Of An Internet-Based Depression Prevention Program: A Randomized Trial Of Primary Care Physician Brief Advice Versus A Motivational Interview. Journal of Cognitive and Behavioral Psychotherapies 2009; 9 (1): 1-19.30. 